\title{
Differential Accumulation of Oil content and Major Fatty Acids During Seed Developmental Stages in Sunflower (Helianthus annuus L.) Hybrids Differing in Oleic Acid Content
}

\author{
Gouspak N. Banuvalli ${ }^{1}$, T.K. Nagarathna ${ }^{2}$, H.G. Praveen ${ }^{1}$ and M. Gayithri ${ }^{1}$ \\ ${ }^{1}$ University of Agricultural Sciences, GKVK, Campus, Bangalore-560065, India \\ ${ }^{2}$ PPV\&FR Authority, Government of India, New Delhi, India \\ *Corresponding author
}

\section{Keywords}

Seed developmental stages, Fatty acids Helianthus annuus Oleic acid content

Article Info

Accepted:

22 June 2020

Available Online:

10 July 2020

\section{A B S T R A C T}

Sunflower (Helianthus annuus L.) is one of the widely cultivated oilseed crops, mainly grown for seed yield and oil content. Its oil contains saturated and unsaturated fatty acids with different biochemical composition which determine the quality and it differs with different hybrids improving the nutritional value for human health. To study their accumulation pattern, research was conducted in six contrasting hybrids differing in oleic acid content (high, mid and low types). During seed development stage, increase in accumulation of oleic acid up to harvest was observed for mid (KBSH-44 \& DRSH-1) and high (PAC 3794 \& RSFH-1) oleic hybrids. In low oleic hybrids (KBSH-71 \& RSFH130) oleic acid content increased up to 16 DAA and decreased thereafter. Linoleic acid increased during seed development only in low types compared to mid and high types. Seed oil content increased with decreased seed moisture. Correlation between oil and unsaturated fatty acid accumulation was different in hybrids. Negative correlation was observed between oil and linoleic acid accumulation in mid and high oleic. In our study, climatic factors did not show any effect on accumulation pattern of major fatty acids in hybrids and it was mainly due to their respective genotypic characters.

\section{Introduction}

Sunflower (Helianthus annuus L.) is one of the widely cultivated oilseed crops in the world with abundant health benefits due to presence of high levels of polyunsaturated fatty acid in the oil. Sunflower seeds known to contain about 24-42 per cent oil (Prolea et $a l ., 2009)$ and is a rich source of unsaturated fatty acids which contributes about 90 percent of total fatty acids. Contribution of oleic acid (monounsaturated fatty acid) was reported to be 12-24 percent and poly unsaturated fatty acid (linoleic acid) is about $60-70$ percent and other fatty acids especially saturated fatty acids contribute for 5-10 percent of total fatty 
acids which are mainly palmitic and stearic acid (Claudio et al., 2014).

Among all the fatty acids, oleic acid is an important monounsaturated fatty acid with a single double bond which can withstand high cooking temperature with low oxidation. Several scientists attempted to develop genotypes with increased oleic acid in many crops such as soybean, sunflower, groundnut etc. (Fernandez et al., 2002, Boersma et al., 2012) for its high stability, improved shelf life and other health benefits. Recent researchers have attempted to develop sunflower hybrids with varying oleic acid concentration and classified them as high oleic, mid oleic and low oleic or standard linoleic hybrids and studied pattern of accumulation of fatty acids during seed development stages (Gesch and Johnson, 2013).

Several available literatures show that accumulation of oleic acid varies during seed development stages in sunflower due to differential expression of stearic acid desaturase (SAD) gene after flowering and fatty acid desaturase (FAD2-1) which increases the activity of linoleic acid as reported by Kabbaj et al., (1996). Hence, to understand the pattern of accumulation and the stage at which these components are accumulated at a maximum level in seeds, the study was conducted in contrasting sunflower hybrids differing in oleic acid.

\section{Materials and Methods}

Six sunflower hybrids were selected based on their oleic acid content in the sunflower seeds and confirmed for their oleic acid content before sowing. Based on their oleic acid content, PAC-3794 (78.03\%) and RSFH-1 $(82.54 \%)$ were classified as high, DRSH$1(52.27 \%)$ and $\mathrm{KBSH}-44(46.27 \%)$ as mid oleic and KBSH-71 (25.71\%) and RSFH-130 $(36.59 \%)$ as low types. The research was carried out at University of Agricultural Sciences, Bangalore during 2017-18 in red sandy clay loam soil with slightly acidic $\mathrm{pH}$. During the experimental period in kharif the $\mathrm{T}_{\max }$ was $28.9^{\circ} \mathrm{C}$ and $\mathrm{T}_{\min } 19.6{ }^{\circ} \mathrm{C}$ with a minimum and maximum rainfall of about 0.4 and $92.4 \mathrm{~mm}$, respectively and total rainfall recorded was $397.6 \mathrm{~mm}$ during crop growth period. Average brightness of sunshine hours was about 4.93 hours recorded during the crop growth period.

Observations on several morphological and physiological parameters (data not shown) were recorded during the crop growth period. From anthesis to maturity at 5 days interval, observations on morphological and yield parameters were recorded. Accordingly, at 5 days interval seeds were collected from the outer three whorls of flower head for estimating oil content and fatty acid composition. The total quantity of seeds obtained from threshing after harvest were dried completely from each selected plant, weighed and expressed in grams to get the final seed yield.

To determine seed moisture content, seed sample of three gram was collected at each stage and made into fine powder with the help of grinder. This fine powder of seed used for estimating seed moisture content using seed moisture meter. Total oil content was estimated using Nuclear Magnetic Resonance (NMR) method. This instrument gives direct values of oil content in the seeds in terms of percentage (\%). Fatty acid composition was determined using crude oil and was extracted by grinding seeds with chloroform: methanol (3:1) and which has been further filtered through Whatmann filter paper and the chloroform: methanol evaporated by keeping it in a hot air oven. The oil from this was used for preparation of fatty acid methyl esters by using $\mathrm{BF}_{3}-\mathrm{Methanol}$ as a reagent at $52^{\circ} \mathrm{C}$ for 2 min, and around $1 \mu l$ of fatty acid methyl ester 
was injected to the gas chromatography 7890A (Plate 6) having column area of $30 \mathrm{~m}$ $\times 0.32 \mathrm{~mm}$ and thickness of 0.25 micron, the column temperature was programmed from 60 to $350{ }^{0} \mathrm{C}$.; where injector and detector temperature set at $250{ }^{0} \mathrm{C}$ using helium, air and hydrogen. The fatty acids were determined based on retention time of the respective standards used, which was carried out for 5 days interval samples from flowering to physiological maturity.

\section{Results and Discussion}

\section{Moisture content}

Moisture content of developing sunflower seeds were decreased during seed developmental stages. Maximum seed moisture was observed at the time of anthesis irrespective of hybrids and high seed moisture content was maintained up to 11 DAA. Differences were observed among the hybrids for seed moisture during seed developmental stages. The lowest seed moisture content for hybrids was observed at the time of harvest, which was in the range of 31.56-46.13 percent. At harvest, low oleic hybrid RSFH130 had higher seed moisture content with 46.13 per cent, followed by high oleic RSFH1 with 42.55 per cent. At harvest $\mathrm{KBSH}-71$ (low oleic) had seed moisture content 37.84 per cent followed by KBSH-44 (mid oleic) with 37.50 per cent. The lowest seed moisture content at harvest was observed for mid oleic hybrid DRSH-1 with 31.56 percent (Table 1).

\section{Seed yield}

Significant difference in seed yield was observed among 6 hybrids (Table 1). Maximum seed yield was found in mid oleic type, KBSH-44 (78.66g) followed by RSFH130 a low oleic with $77.07 \mathrm{~g}$ which was on par with the DRSH-1 (mid oleic) with $70.87 \mathrm{~g}$. Low oleic hybrid KBSH-71 had seed yield of
$66.37 \mathrm{~g}$ per plant. Lowest seed yield were obtained for high oleic types RSFH-1 and PAC-3794 with 61.26 and $56.24 \mathrm{~g}$ per plant respectively.

\section{Crude oil}

Oil content was found to increase from anthesis to physiological maturity. Seed oil content was found to differ between hybrids during seed developmental stages. Oil accumulation during seed development was low up to 11 DAA and increased thereafter upto 31 DAA in most hybrids except for KBSH-71 (low oleic) and DRSH-1 (mid oleic) which has stabilized oil accumulation at 26 DAA itself. PAC-3794 (high oleic) was known to accumulate oil at faster rate and reaches 31.58 per cent at 21 DAA and increased slightly thereafter to reach 34.00 per cent at maturity. The higher oil content at the time of harvest was observed for the hybrids KBSH-71 (low oleic) and KBSH-44 (mid oleic) with mean values of 35.59 and 35.00 per cent respectively, followed by DRSH-1 (34.70\%) and PAC-3794 (34.00\%). Lowest oil content was observed for high oleic hybrid RSFH-1 with 27.55 per cent at harvest. For all the hybrids the accumulation of oil was at faster rate between 16-31 days after anthesis of seed developmental stages except for high oleic hybrid PAC-3794 between 11-21 DAA.

\section{Oleic acid (\%)}

More variation for accumulation of oleic acid has been found for the low-oleic hybrids i.e. KBSH-71 and RSFH-130 which had a range of 20.93-46.21 and 15.51-35.12 per cent, respectively during seed development stages (Table 2). In low oleic hybrids, increase in oleic acid has been found up to 16 DAA for KBSH-71 with the value of 46.21 per cent and up to 21 DAA for RSFH-130 with value of 35.12 per cent and further oleic acid content decreased thereafter to reach 32.70 
and 31.71 per cent at maturity for KBSH-71 and RSFH-130, respectively. Higher percentage of oleic acid at the time of harvest was observed for the hybrids PC-3794 and RSFH-1 with 83.90 and 73.53 per cent, respectively. In mid oleic hybrids, oleic acid per cent during seed developmental stages was found to be in the range of 31.89-68.07 per cent for KBSH-44 and 25.23-63.34 for DRSH-1. For mid oleic hybrids the oleic acid percentage was found to be increased during seed developmental stages reaching to maximum at maturity.

\section{Linoleic acid (\%)}

Linoleic acid percentage was found to increase during seed developmental stages for low oleic types KBSH-71 (21.55\%) and RSFH-130 (16.87\%) at anthesis, reaching maximum at 31 days after anthesis (physiological maturity) with 51.17 and 49.67 per cent, respectively (Table 2). In mid oleic types the linoleic acid percentage during seed developmental stages was reported to be in the range of 16.23-39.03 per cent for KBSH44 and 20.65-31.90 per cent for DRSH-1. In mid oleic types, linoleic acid was increased up to 11 DAA and 16 DAA for KBSH-44 (39.03 per cent) and DRSH-1 (31.90 per cent). In high oleic types, PAC-3794 and RSFH-1, linoleic acid accumulation was more at 6 DAA with values of 21.93 and 38.52 per cent respectively. In high oleic hybrids, linoleic acid during seed developmental stages was found to be in the range of 3.9021.93 and 5.39-38.52 per cent for PAC-3794 and RSFH-, 1 respectively. For high oleic types, linoleic acid percentage decreased from 6 DAA to reach minimum values at physiological maturity.

More accumulation of linoleic acid was on 16 DAA for low oleic (KBSH-71 and RSFH130) hybrids, as expression of FAD2-1 was reported after the 18 days of flowering
(Kabbaj et al., 1995, Lagravare et al., 2004 and Onmeli, 2012) in low oleic types. Linoleic acid in mid oleic types has found to increase upto 11 DAA (KBSH-44) and 16 DAA (DRSH-1) that might be due to lesser activity of FAD2-1 enzyme and further decrease was due to the dilution effect of newly synthesized fatty acid i.e. oleic acid. Differences among the mid oleic types varied little, which may be due to their genetic makeup i.e. dependent on the stability of the parental lines for oleic acid across environment used for developing respective hybrids. In high oleic hybrids (PAC-3794 and RSFH-1) accumulation of linoleic acid was decreased from 6 DAA might be due to no enzyme activity of FAD2-1 high oleic types. The activity of FAD2-1 is found only upto 12 days after the end of flowering in the high oleic genotypes.

\section{Palmitic acid (\%)}

Palmitic acid was decreased during the seed developmental stages and higher accumulation at the time of anthesis for all hybrids. Higher palmitic acid accumulation at the time of anthesis was found in high oleic RSFH-1 (39.83\%), followed by low oleic KBSH-71 (36\%), KBSH-44 (mid oleic) with 31.91 per cent and RSFH-130 (low oleic) with 29 per cent and lower palmitic acid observed for high oleic PAC-3794 (14.34\%).

In all the six hybrids the decrease in palmitic acid during seed developmental stages was due to increase in accumulation of other major long chain fatty acids (oleic and linoleic acid).

Higher range was found to be more in standard hybrids than in mid-oleic and higholeic as these differences was due to genotype where accumulation of palmitic acid was known to be less influenced by the environmental conditions. 


\section{Stearic acid}

Stearic acid for low oleic types, KBSH-71 and RSFH-1 was found to be in the range of 3.57-11.85 and 3.62-17.43 per cent, respectively. For all the hybrids, stearic acid content was found maximum at anthesis itself and further found to decrease during seed development till maturity. During seed developmental stages, mid oleic hybrids had stearic acid content in the range of 3.84-8.59 and 5.09-8.27 per cent for KBSH-44 and DRSH-1, respectively. For high oleic hybrids, PAC-3794 and RSFH-1 stearic acid found in the range of $3.50-8.07$ and $3.44-10.55$ per cent, respectively during seed development. Stearic acid content varied little during seed development stages within hybrid, which was reached to lower levels at maturity. At harvest stearic acid content was highest for low oleic hybrid KBSH-71 (7.15\%), followed by mid oleic hybrid KBSH-44 (5.57\%) and lowest stearic acid percentage was observed for high oleic hybrid PAC-3794 (3.50\%).

\section{Minor fatty acid composition}

In sunflower 19-21 minor fatty acids excluding the four major fatty acids (palmitic, stearic, oleic and linoleic acid) are found at 1 DAA (data not shown). The minor fatty acids were methyl undecanoate, methyl laurate, methyl tridecanoate, methyl myristate, myristoleate methyl ester, elaidic acid methyl ester, lenolelaidic acid methyl ester, methyl linoleate, methyl arachidate, gamma linoliec acid methyl ester, methyl eicosanoate, methyl heneicosanoate, methyl eicosadienoate, methyl behanatecis-11,14,17-eicotrienoic acid methyl ester, methyl cis- 5,8,11,14,17 eicosapentaenoate, methyl tricosanoate, cis 13,16- docosadienoic acid methyl ester, methyl lignocerate, methyl cis- 5,8,11,14,17 eicosapentaenoate, methyl nervonate, These fatty acids contribute around 14-23 percent to that of total fatty acids at 1 DAA. These fatty acids decreased thereafter during seed developmental stages and were undetectable at maturity. Differences in the percentage of these minor fatty acids were observed among the hybrids.

\section{Correlation study between fatty acids and with oil content}

\section{Oleic acid and Linoleic acid}

Trend of accumulation of unsaturated fatty acids (oleic acid and linoleic acid) is represented in the Fig. 1. Accumulation of oleic acid differed for hybrids during seed development. In low oleic hybrids KBSH-71 and RSFH-130, linoleic acid increased during seed development stages. Oleic acid increased up to 16 DAA and 21 DAA for KBSH-71 and RSFH-130 respectively, which decreased thereafter till maturity. Oleic acid for mid oleic hybrids KBSH-44 and DRSH-1 has increased up to 11 DAA and 16 DAA respectively and decreased thereafter till maturity, whereas linoleic acid increased for both the hybrids during seed development. In high oleic types (PAC-3794 and RSFH-1), higher linoleic acid was observed at 6 DAA and decreased thereafter till maturity and oleic acid content increased during seed development.

\section{Oil content and Oleic acid}

Accumulation of oleic acid differed in hybrids, whereas oil content increased during seed development irrespective of type of hybrids (Fig.2). However, oil content varied among the hybrids during seed development. Among contrasting hybrids, oleic acid content increased with increase in oil content upto 16 DAA and thereafter oleic acid decreased with the increase in production of linoleic acid. In high and mid oleic types oleic acid content increased with the increase in oil content up to physiological maturity. 
Table.1 Seed moisture (\%), seed oil content (\%) at different seed developmental stages of sunflower hybrids and seed yield (g) at harvest

\begin{tabular}{|c|c|c|c|c|c|c|c|c|c|c|c|c|c|c|c|c|}
\hline \multirow[t]{2}{*}{ Oleic type } & \multirow[t]{2}{*}{ Hybrids } & \multicolumn{7}{|c|}{ Seed moisture (Days after anthesis) } & \multicolumn{7}{|c|}{ Seed oil content (Days after anthesis) } & \multirow{2}{*}{$\begin{array}{c}\text { Seed } \\
\text { yield }(\mathrm{g})\end{array}$} \\
\hline & & 1 & 6 & 11 & 16 & 21 & 26 & 31 & 1 & 6 & 11 & 16 & 21 & 26 & 31 & \\
\hline \multirow{2}{*}{$\begin{array}{l}\text { High oleic } \\
\text { hybrids }\end{array}$} & PAC-3794 & 87.69 & 88.94 & 81.90 & 76.51 & 61.85 & 58.67 & 37.43 & 4.39 & 6.20 & 12.11 & 17.61 & 31.58 & 32.38 & 34.00 & 56.24 \\
\hline & RSFH-1 & 88.48 & 88.16 & 83.54 & 77.63 & 65.45 & 57.44 & 55 & 2.00 & 6.17 & 11.65 & & & 26.65 & & \\
\hline \multirow{2}{*}{$\begin{array}{c}\text { Mid oleic } \\
\text { hybrids }\end{array}$} & KBSH-44 & & 86.33 & 81.92 & 76.66 & 60.37 & 57.86 & & 1.73 & 3.17 & 10.86 & & 23.05 & 26.96 & 35 & 78.66 \\
\hline & DRSH-1 & 85.84 & 87.87 & 84.15 & 78.29 & 62.89 & 60.60 & 31.56 & 0.52 & 7.42 & 8.98 & 13.30 & 21.34 & 31.34 & 34.70 & 70.87 \\
\hline \multirow{2}{*}{$\begin{array}{l}\text { Low oleic } \\
\text { hybrids }\end{array}$} & KBSH-71 & 87.39 & 88.64 & 84.09 & 73.63 & 67.19 & 57.20 & 37.84 & 1.08 & 5.18 & 9.96 & 15.79 & 25.48 & 35.35 & 35.59 & 66.37 \\
\hline & RSFH-130 & 87.80 & 85.92 & 81.21 & 70.92 & 74.92 & 63.97 & 46.13 & 0.52 & 3.22 & 9.29 & 14.92 & 20.91 & 23.81 & 30.54 & 77.07 \\
\hline
\end{tabular}

Table.2 Mean values of oleic and linoleic acid in the developing seeds of sunflower hybrid

\begin{tabular}{|c|c|c|c|c|c|c|c|c|c|c|c|c|c|c|c|}
\hline \multirow{2}{*}{$\begin{array}{l}\text { Oleic } \\
\text { type }\end{array}$} & \multirow[t]{2}{*}{ Hybrids } & \multicolumn{7}{|c|}{ Oleic acid (days after anthesis) } & \multicolumn{7}{|c|}{ Linoleic acid (days after anthesis) } \\
\hline & & 1 & 6 & 11 & 16 & 21 & 26 & 31 & 1 & 6 & 11 & 16 & 21 & 26 & 31 \\
\hline \multirow{2}{*}{$\begin{array}{l}\text { High } \\
\text { oleic }\end{array}$} & PAC-3794 & 41.14 & 50.87 & 62.76 & 70.89 & 73.98 & 76.29 & 83.90 & 20.08 & 21.93 & 16.73 & 9.11 & 7.93 & 7.38 & 3.90 \\
\hline & RSFH-1 & 31.17 & 43.31 & 53.87 & 60.15 & 63.92 & 65.71 & 73.53 & 12.53 & 38.52 & 21.47 & 16.49 & 12.98 & 11.12 & 5.39 \\
\hline \multirow{2}{*}{$\begin{array}{l}\text { Mid } \\
\text { oleic }\end{array}$} & KBSH-44 & 31.89 & 37.24 & 45.81 & 58.24 & 65.79 & 66.60 & 68.07 & 22.44 & 35.34 & 39.03 & 23.31 & 20.40 & 18.55 & 16.23 \\
\hline & DRSH-1 & 25.23 & 32.82 & 39.66 & 45.70 & 52.54 & 62.85 & 63.34 & 20.65 & 24.32 & 29.79 & 31.90 & 29.35 & 24.81 & 22.65 \\
\hline \multirow{2}{*}{$\begin{array}{l}\text { Low } \\
\text { oleic }\end{array}$} & KBSH-71 & 20.93 & 28.02 & 35.55 & 46.21 & 38.76 & 35.07 & 32.70 & 21.55 & 24.12 & 26.27 & 30.55 & 36.33 & 46.00 & 51.17 \\
\hline & RSFH-130 & 15.51 & 23.23 & 27.30 & 30.96 & 35.12 & 34.41 & 31.71 & 16.87 & 27.65 & 34.78 & 40.24 & 45.74 & 48.04 & 49.67 \\
\hline
\end{tabular}

Table.3 Mean values of palmitic (\%) and stearic acid (\%) in the developing seeds of sunflower hybrids

\begin{tabular}{|c|c|c|c|c|c|c|c|c|c|c|c|c|c|c|c|}
\hline \multirow{2}{*}{$\begin{array}{l}\text { Oleic } \\
\text { type }\end{array}$} & \multirow[t]{2}{*}{ Hybrids } & \multicolumn{7}{|c|}{ Palmitic acid (days after anthesis) } & \multicolumn{7}{|c|}{ Stearic acid (days after anthesis) } \\
\hline & & 1 & 6 & 11 & 16 & 21 & 26 & 31 & 1 & 6 & 11 & 16 & 21 & 26 & 31 \\
\hline \multirow{2}{*}{$\begin{array}{l}\text { High } \\
\text { oleic }\end{array}$} & PAC-3794 & 14.34 & 15.81 & 13.49 & 12.60 & 12.12 & 10.85 & 5.88 & 8.07 & 7.17 & 5.84 & 5.88 & 5.82 & 6.92 & 3.50 \\
\hline & RSFH-1 & 39.83 & 15.51 & 14.66 & 11.66 & 11.40 & 8.05 & 6.21 & 3.44 & 4.49 & 3.98 & 10.55 & 7.68 & 5.53 & 3.73 \\
\hline \multirow{2}{*}{$\begin{array}{l}\text { Mid } \\
\text { oleic }\end{array}$} & KBSH-44 & 31.91 & 18.90 & 10.01 & 8.91 & 7.22 & 6.84 & 4.82 & 3.84 & 4.39 & 5.35 & 8.59 & 6.73 & 6.56 & 5.57 \\
\hline & DRSH-1 & 27.67 & 26.65 & 23.87 & 13.10 & 10.29 & 6.66 & 7.12 & 5.66 & 5.17 & 5.30 & 8.27 & 7.73 & 5.81 & 5.09 \\
\hline \multirow{2}{*}{$\begin{array}{l}\text { Low } \\
\text { oleic }\end{array}$} & KBSH-71 & 36.00 & 28.90 & 23.76 & 14.85 & 11.20 & 7.20 & 6.40 & 3.57 & 11.85 & 10.28 & 8.41 & 6.64 & 8.48 & 7.15 \\
\hline & RSFH-130 & 29.00 & 25.78 & 24.60 & 19.80 & 13.12 & 8.08 & 5.83 & 17.43 & 15.73 & 10.55 & 9.18 & 6.07 & 4.77 & 3.62 \\
\hline
\end{tabular}




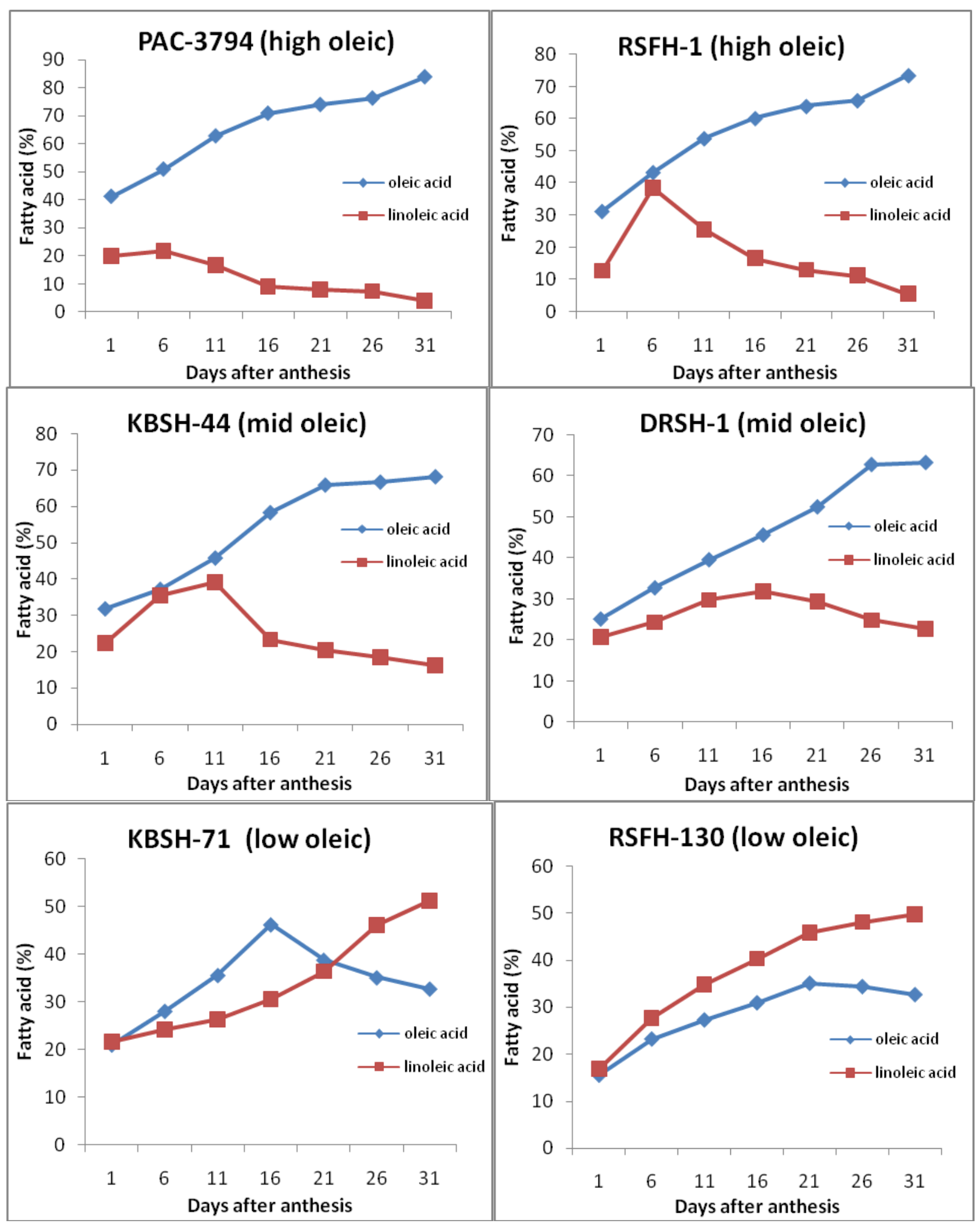

Fig.1 Accumulation of oleic and linoleic acid from anthesis to physiological maturity of contrasting sunflower hybrids 


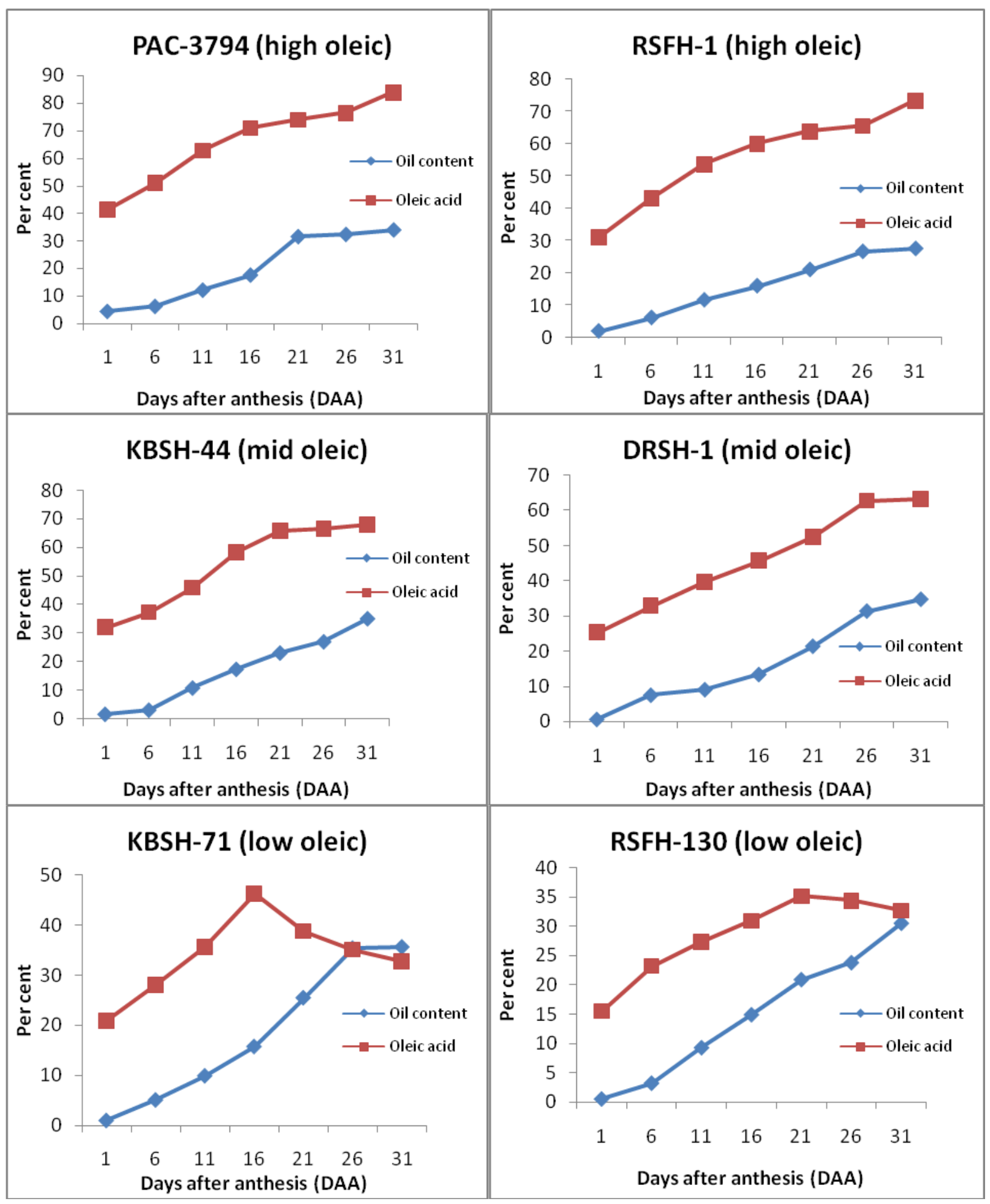

Fig.2 Accumulation of oil content and oleic acid from anthesis to physiological maturity of contrasting sunflower hybrids 


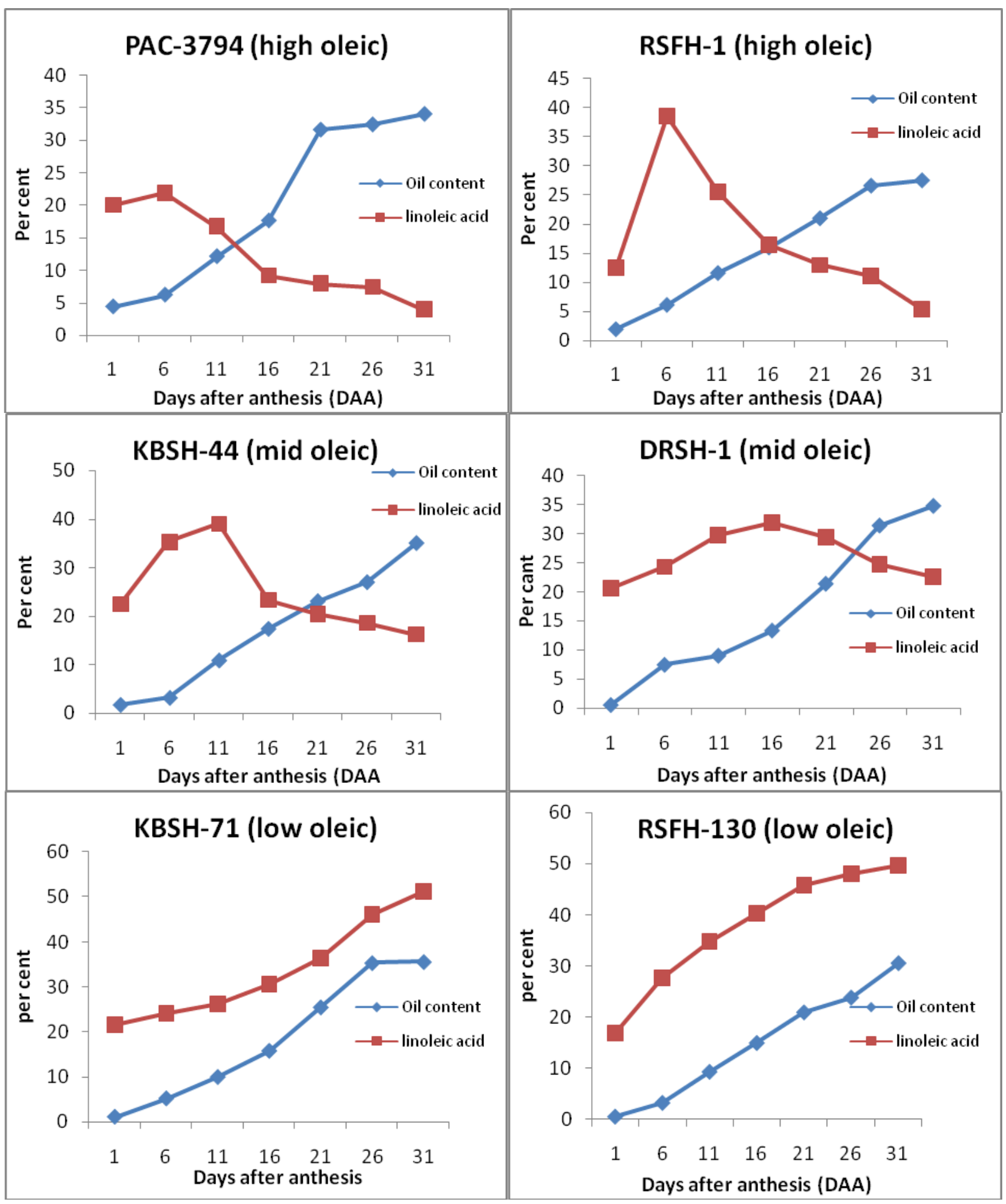

Fig.3 Accumulation of oil and linoleic acid of contrasting sunflower hybrids during seed development 


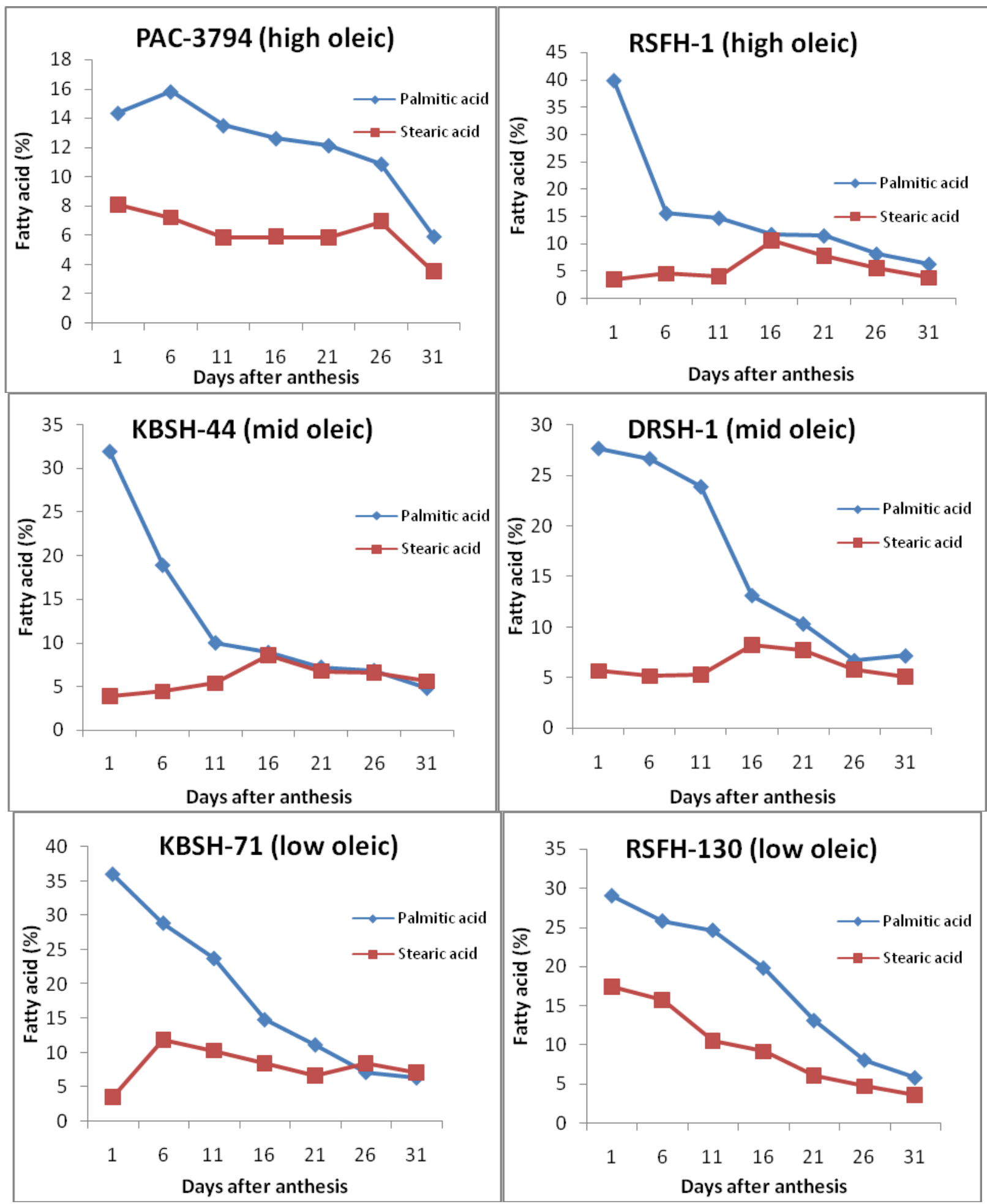

Fig.4 Accumulation of palmitic and stearic acid of contrasting sunflower hybrids during seed development 


\section{Oil content and Linoleic acid}

During seed development in sunflower accumulation of oil increased with increase in linoleic acid in the low oleic hybrids (KBSH71 and RSFH-130) up to physiological maturity (Fig. 3). Mid oleic acid had increased linoleic acid with the increase in oil content up to 11 DAA and decreased thereafter. In high oleic types, linoleic acid increased with increase in oil content up to 6 DAA and decreased thereafter.

\section{Palmitic acid and Stearic acid}

Palmitic and stearic acids are the two saturated fatty acids which have been found to be around 12-15 per cent of total fatty acids of sunflower seeds at harvest. During seed development the accumulation of these saturated fatty acids differed. In low oleic hybrids (RSFH-130) both palmitic and stearic acid content decreased during seed development and for KBSH-71 palmitic acid decreased during seed development, whereas stearic acid increased up to 6 DAA and decreased thereafter till maturity. In mid oleic (KBSH-44 and DRSH-1) hybrids increase in stearic acid was found up to $16 \mathrm{DAA}$ and decreased thereafter, whereas, palmitic acid decreased during seed development for both the hybrids. In high oleic hybrids (PAC-3794 and RSFH-1) palmitic acid decreased during seed development, whereas stearic acid accumulation in RSFH-1 increased upto 16 DAA and decreased thereafter till maturity and in PAC-3794 stearic acid content varied during seed development (Fig. 4).

\section{Oil content (\%)}

A faster rate of accumulation of oil between 16-26 days after anthesis and stabilized till physiological maturity with slight increase may be due to incorporation of fatty acids for triacylglycerol synthesis which takes place only after the 12 DAA and reaches maximum at 25 DAA (Garces and Mancha., 1991).

\section{Oleic acid (\%)}

Variation was more for the standard hybrids (low oleic hybrids) due to interaction of genotype with environment. Increase in oleic acid at initial stages up to 16-21 DAA might be due to increase in expression of stearic acid desaturase (SAD) gene between 10-20 days after flowering. Further decrease in oleic acid after 21 days of anthesis was certainly due to the formation of linoleic acid by increase in activity of fatty acid desaturase (FAD2-1). Differences in oleic acid accumulation among the mid oleic and high oleic types might be due to their differences in FAD2-1 activity which is dependent on genetic characters (Kabbaj et al., 1995).

\section{Linoleic acid (\%)}

More accumulation of linoleic acid was on 16 DAA for low oleic (KBSH-71 and RSFH130) hybrids, as expression of FAD2-1 was reported after the 18 days of flowering (Kabbaj et al., 1995 and Onmeli, 2012) in low oleic types. Linoleic acid in mid oleic types has found to increase upto 11 DAA (KBSH44) and 16 DAA (DRSH-1) that might be due to lesser activity of FAD2-1 enzyme and further decrease was due to the dilution effect of newly synthesized fatty acid i.e. oleic acid. Differences among the mid oleic types varied little, which may be due to their genetic makeup i.e. dependent on the stability of the parental lines for oleic acid across environment used for developing respective hybrids. In high oleic hybrids (PAC-3794 and RSFH-1) accumulation of linoleic acid was decreased from 6 DAA might be due to no enzyme activity of FAD2-1 high oleic types. The activity of FAD2-1 is found only up to 12 days after the end of flowering in the high oleic genotypes (Lagravere et al., 2004) 
which lower linoleic acid accumulation up to physiological maturity.

\section{Palmitic acid (\%)}

Palmitic acid content decreased during the seed developmental stages and higher accumulation at the time of anthesis for all hybrids. In all the six hybrids the decrease in palmitic acid during seed developmental stages was due to increase in accumulation of other major long chain fatty acids (oleic and linoleic acid). Higher range was found to be more in standard hybrids than in mid-oleic and high-oleic as these differences was due to genotype where accumulation of palmitic acid was known to be less influenced by the environmental conditions (Izquierdo et al., 2002 and Zlatanov et al., 2009).

\section{Stearic acid (\%)}

Maximum stearic acid was found at anthesis and decreased during seed developmental stages reaching minimum at physiological maturity (harvest). Decrease in stearic acid during seed development was due to accumulation of long chain unsaturated fatty acids where stearic acid acts as precursor. Differences among the oleic hybrids for stearic acid content during seed development and within the oleic types i.e. among low, mid and high oleic was due to differences in the genotypic character (Onmeli, 2012, Zlatanov et al., 2009 and Izquierdo et al., 2002).

\section{Minor fatty acids}

The accumulation of minor fatty acids was less than 5 per cent at 1 DAA in all six hybrids. This was due to beginning of fatty acids pathway during initial stages of seed formation and 70-80 per cent major fatty acids (palmitic, stearic, oleic and linoleic acid) due to which minor fatty acids are found to accumulate rest of the $15-20$ per cent fatty acids. These minor fatty acids were decreased thereafter during seed development in sunflower due to increase in accumulation of oil and major fatty acids (Onmeli, 2012).

In conclusion, change in fatty acid accumulation during seed development, dependent on the genotypic constitution of each hybrid. Oleic acid accumulation was negatively related to linoleic acid in all the hybrids, whereas similar trend not observed between saturated fatty acids. In high and mid oleic sunflower hybrids, though the linoleic acid content increased at the beginning of anthesis, it declined at the later stages of seed development. However similar trend was not recorded in low oleic hybrids. Irrespective of hybrids, palmitic and stearic acid content was higher during and later on declined at maturity. In all the oleic groups, oil content and oleic acid exhibited positive correlation and both increased from anthesis and reaching maximum at harvest.

\section{References}

Boersma, J, G., Gillman, J, D., Bilyeu, K. D., Ablett, G. R., Grainger, C. and Rajcan, I. 2012. New mutations in a delta-9stearoyl-acp desaturase gene associated with enhanced stearic acid levels in soybean seed. Crop Sci., 52: 1736-1742 Claudio, F., Turi, M. and Vannozzi, G. P. 2014. Variability of seed fatty acid composition to growing degree days in high oleic acid sunflower genotypes. Helia, pp: 1-16

Fernandez-Moya, V., Martinez-Force, E. and Garces, R. 2002. Temperature effect on a high stearic acid sunflower mutant. Phytochem., 59 (1): 33-37

Garces, R. and Mancha, M. 1991. In vitro oleate desaturase in developing sunflower seeds, Phytochem., 30: 21272130

Gesch, R. W. and Johnson, B. L. 2012. Seed 
moisture at physiological maturity in oilseed and confectionary sunflower hybrids in the northern U.S. Field Crops Res., 133: 1-9

Izquierdo, N., L. Aguirrezabal, F. A. and Pereyra, V. 2002. Night temperature affects fatty acid composition in sunflower oil depending on the hybrid and the phenological stage. Field Crops Res., 77(2-3): 115-26

Kabbaj, A., Abbott, A. G., Vervoort, V. and Berville, A. 1995. Expression of stearate, oleate and linoleate desaturase genes in sunflower with normal and high oleic contents. In: Sunflower biotechnology. Eur. Symp., Ebernburg, DEU

Lagravère, T, Kleiber, D, Surel, O , Calmon, A, Bervillé, A, Dayde, J. 2004. Comparison of Fatty Acid Metabolism of Two Oleic and One Conventional Sunflower Hybrids: A New Hypothesis. J. Agron. Crop Sci., 190 (4): 223-229

Onmeli, F., 2012. Changes in oil fatty acid composition during seed development of sunflower. Asi. J. Pl. Sci., 11:241245

Prolea, 2009. The French sector of vegetable oils and proteins, available at: http://www.prolea.com

Zlatanov, M. D., Angelova-Romova, M. J., Antova, G. A., Dimitrova, R. D., Momchilova, S. M. and Boryana, M. N. 2009. Variations in fatty acids, phospholipids and sterols during the seed development of a high oleic sunflower variety. $J$ Amer. Oil Chemists' Soci., 86 (9): 867-875.

\section{How to cite this article:}

Gouspak N. Banuvalli, T.K. Nagarathna, H.G. Praveen and Gayithri, M. 2020. Differential Accumulation of Oil content and Major Fatty Acids During Seed Developmental Stages in Sunflower (Helianthus annuus L.) Hybrids Differing in Oleic Acid Content. Int.J.Curr.Microbiol.App.Sci. 9(07): 3216-3228. doi: https://doi.org/10.20546/ijcmas.2020.907.375 